\title{
Extracranial Carotid and Vertebral Artery Dissection: A Review
}

\author{
Gary John Redekop
}

\begin{abstract}
Dissection of the extracranial carotid and vertebral arteries is increasingly recognized as a cause of transient ischemic attacks and stroke. The annual incidence of spontaneous carotid artery dissection is 2.5 to 3 per 100,000 , while the annual incidence of spontaneous vertebral artery dissection is 1 to 1.5 per 100,000 . Traumatic dissection occurs in approximately $1 \%$ of all patients with blunt injury mechanisms, and is frequently initially unrecognized. Overall, dissections are estimated to account for only $2 \%$ of all ischemic strokes, but they are an important factor in the young, and account for approximately $20 \%$ of strokes in patients less than 45 years of age. Arterial dissection can cause ischemic stroke either by thromboemboli forming at the site of injury or as a result of hemodynamic insufficiency due to severe stenosis or occlusion. Available evidence strongly favors embolism as the most common cause. Both anticoagulation and antiplatelet agents have been advocated as treatment methods, but there is limited evidence on which to base these recommendations. A Cochrane review on the topic of antithrombotic drugs for carotid dissection did not identify any randomized trials, and did not find that anticoagulants were superior to antiplatelet agents for the primary outcomes of death and disability. Healing of arterial dissections occurs within three to six months, with resolution of stenosis seen in $90 \%$, and recanalization of occlusions in as many as 50\%. Dissecting aneurysms resolve on follow-up imaging in 5-40\%, decrease in size in 15-30\%, and remain unchanged in 50-65\%. Resolution is more common in vertebral dissections than in carotid dissections. Aneurysm enlargement occurs rarely. The uncommon patient presenting with acute hemodynamic insufficiency should be managed with measures to increase cerebral blood flow, and in this setting emergency stent placement to restore cerebral perfusion may be considered, provided that irreversible infarction has not already occurred.
\end{abstract}

RÉSUMÉ: Dissection de la portion extracrânienne de la carotide et de l'artère vertébrale : revue de la littérature. On reconnaît de plus en plus que la dissection de la portion extracrânienne de la carotide et de l'artère vertébrale peut être une cause d'ischémie cérébrale transitoire (ICT) et d'accident vasculaire cérébral (AVC). L'incidence annuelle de la dissection spontanée de la carotide est de 2,5 à 3 par 100,000 alors qu'elle est de 1 à 1,5 par 100,000 pour la dissection spontanée de l'artère vertébrale. La dissection traumatique survient chez à peu près $1 \%$ de tous les patients qui subissent une contusion et passe souvent inaperçue lors de l'évaluation initiale. On estime que les dissections sont responsables de seulement $2 \%$ de tous les accidents ischémiques, mais elles constituent un facteur important chez les jeunes et elles sont la cause d'à peu près $20 \%$ des accidents vasculaires chez les patients de moins de 45 ans. La dissection artérielle peut causer un accident ischémique soit par thromboembolie à l'endroit du traumatisme ou à cause de l'insuffisance hémodynamique due à une sténose sévère ou à une occlusion. Selon les données actuelles, la cause la plus fréquente serait l'embolie. Les anticoagulants et les antiplaquettaires ont été proposés comme traitement, mais il existe peu de données pour étayer ces recommandations. Une revue systématique (Cochrane Library) portant sur l'administration d'antithrombotiques dans la dissection carotidienne n'a pas identifié d'essai randomisé et n'a pas déterminé si les anticoagulants étaient supérieurs aux agents antiplaquettaires quand le critère d'évaluation principal était le décès et l'invalidité. Une dissection artérielle guérit en 3 à 6 mois, avec résolution de la sténose dans $90 \%$ des cas et recanalisation de l'occlusion dans $50 \%$ des cas. Le suivi par imagerie des anévrismes disséquant démontre une résolution dans 5 à $40 \%$ des cas, une diminution de la taille de l'anévrisme dans 15 à $30 \%$ des cas et aucun changement dans 50 à $65 \%$ des cas. La résolution est plus fréquente dans les dissections vertébrales que dans les dissections carotidiennes. On observe rarement une augmentation de la taille de l'anévrisme. Chez les rares patients qui présentent une insuffisance hémodynamique aiguë, le traitement en phase aiguë vise à augmenter la perfusion cérébrale et on peut envisager la mise en place d'un stent d'urgence en l'absence d'infarctus irréversible.

Can. J. Neurol. Sci. 2008; 35: 146-152

From the Division of Neurosurgery, Vancouver General Hospital, The University of British Columbia, Vancouver, British Columbia, Canada.

Received August 16, 2007. Final Revisions Submitted November 13, 2007.

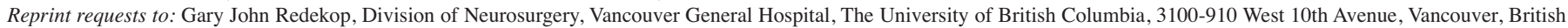
Columbia, V5Z 4E3, Canada. 
Dissection of the extracranial carotid and vertebral arteries is increasingly recognized as a cause of transient ischemic attacks and stroke. ${ }^{1,2}$ The annual incidence of spontaneous carotid artery dissection is 2.5 to 3 per 100,000 , while the annual incidence of spontaneous vertebral artery dissection is 1 to 1.5 per 100,000 . Overall, these conditions are estimated to account for only $2 \%$ of all ischemic strokes, but they are an important factor in the young, and account for approximately $20 \%$ of strokes in patients less than 45 years of age. ${ }^{3}$

Although recent developments in non-invasive neurovascular imaging have led to growing awareness of this complex problem, the highly variable course of cervical artery dissection still poses a major challenge for the treating physician. Recent publications in the neurology literature have described the clinical features of spontaneous dissection, risk factors, and treatment considerations. ${ }^{4-10}$ The Canadian Stroke Consortium reported a non-randomized pilot study comparing anticoagulation and aspirin in 116 patients with carotid or vertebral dissection, and found no significant difference in stroke rates. They calculated that a randomized trial comparing the two treatments would require almost 2,000 patients in order to demonstrate an absolute risk reduction of $5 \% .{ }^{11}$

Most publications in the neurological literature have focused on "spontaneous" dissection as a cause of stroke in the young, and have specifically excluded patients with "traumatic" dissection. However, there is no obvious pathological difference between dissections occurring spontaneously, after minor trauma, or as a result of more severe mechanisms of injury. ${ }^{3} \mathrm{~A}$ history of minor trauma or activity associated with hyperextension or rotation of the neck, such as practicing yoga, painting a ceiling or coughing is frequently obtained in patients with so-called spontaneous dissection. In practice, dissections are typically labeled spontaneous in the absence of major blunt injury or acceleration - deceleration mechanisms associated with craniocervical trauma, and usually present with symptoms of cerebrovascular ischemia. Debate has largely focused on the best treatment to prevent recurrent stroke in patients diagnosed with dissection after they have already suffered an ischemic event. ${ }^{1-}$ 3,5-13 Spontaneous and post-traumatic dissection less frequently involves the intracranial circulation, where the risk of hemorrhage is also a factor in presentation and management. This review is limited to dissection affecting only the extracranial carotid and vertebral arteries.

Concurrent with recent developments in the diagnosis and treatment of "spontaneous" dissection, widely available noninvasive neurovascular screening studies such as MR and CT angiography have revealed an epidemic of carotid and vertebral dissections in patients with blunt trauma. The devastating potential of blunt vascular injuries, and the importance of early recognition and appropriate therapy to prevent ischemic cerebrovascular complications, is increasingly appreciated. ${ }^{14-19}$ The benefit in terms of stroke prevention from the identification and treatment of traumatic carotid and vertebral dissections before they lead to cerebral ischemia may be substantial, and neurologists should take an interest and active role in the management of patients with traumatic cerebrovascular injuries.

\section{Pathology}

Dissections of the extracranial carotid and vertebral arteries arise from tears that occur in the intimal layer, allowing blood under arterial pressure to enter the wall of the vessel and form an intramural hematoma. Disruption of the endothelium can activate platelets and the coagulation cascade, leading to thrombus formation and distal embolization. If the major dissection plane is between the intima and media, the intramural hematoma may expand the arterial wall enough to cause compression of the normal lumen diameter with severe stenosis or occlusion causing ischemic symptoms or stroke due to hemodynamic insufficiency (Figure 1). Alternatively, if the dissection tracks between the media and adventitia, an aneurysmal outpouching can result. These dissecting aneurysms can cause symptoms of mass effect and compression of the lower cranial nerves below the skull base, or can be a source of thromboemboli (Figure 2). ${ }^{2,3}$

\section{Spontaneous Dissections}

Patients with spontaneous dissections may have underlying structural defects of the arterial wall, including connective tissue disorders such as Ehlers-Danlos syndrome type 4, Marfan's syndrome, polycystic kidney disease, and osteogenesis imperfecta type $\mathrm{I}^{3}$ However, there is frequently a history of minor trauma with hyperextension or rotation of the neck. These movements, especially when sudden, may injure the artery as a result of mechanical stretching of the mobile segments of the extracranial vessels.

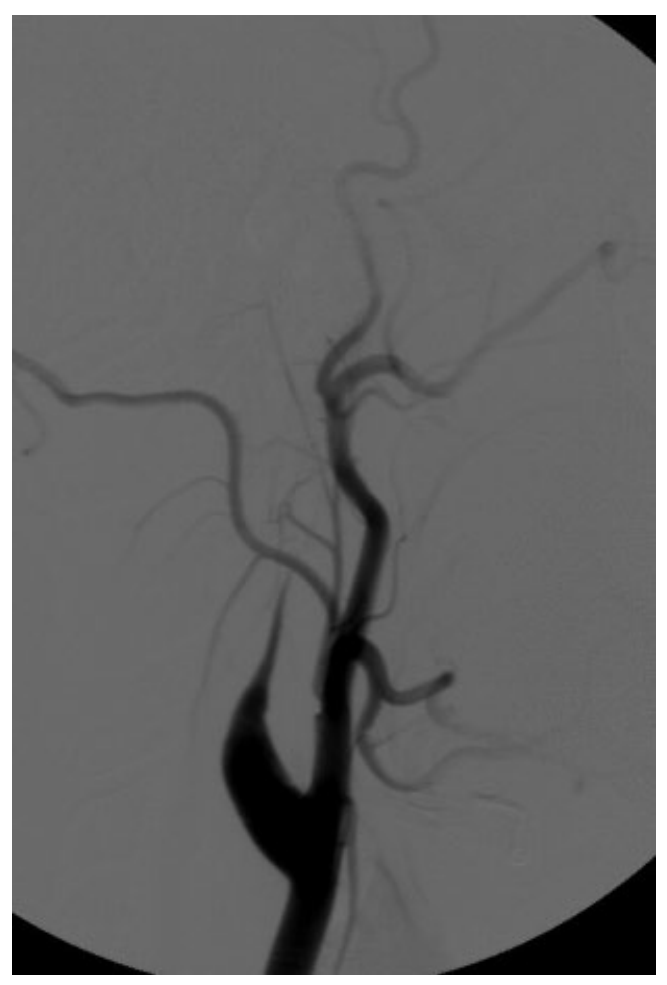

Figure 1: Lateral cervical carotid angiogram showing typical carotid dissection with flame-shaped narrowing tapering to occlusion, beginning 2 to $3 \mathrm{~cm}$ distal to the carotid bulb. 


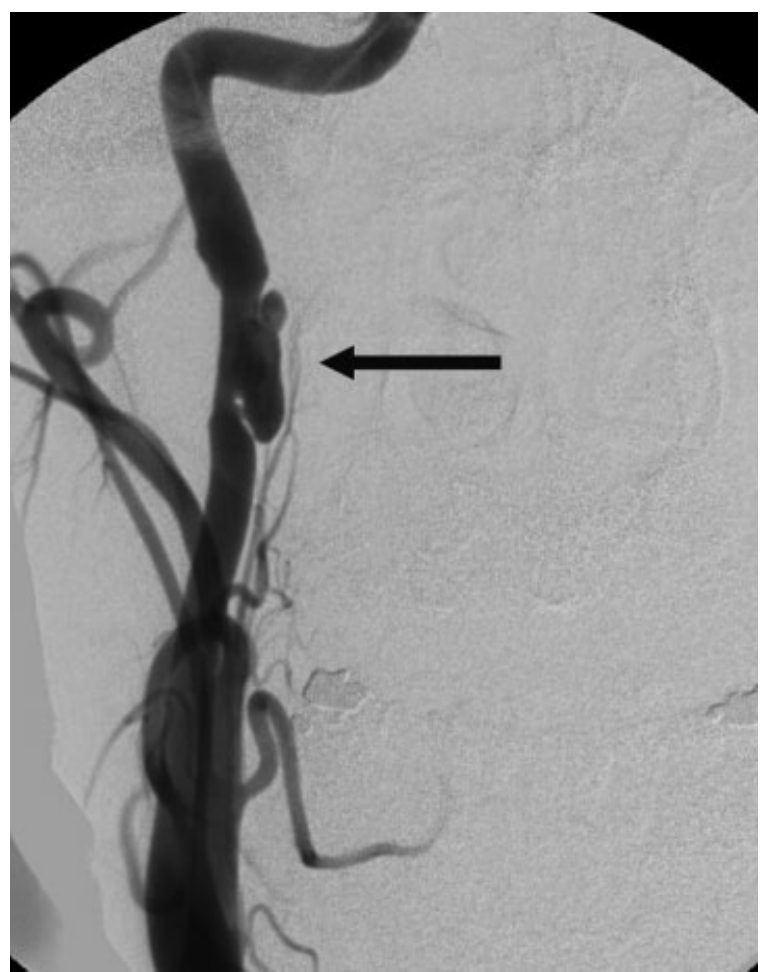

Figure 2: Anterior-posterior cervical carotid angiogram showing dissection of the distal extracranial internal carotid artery with aneurysm formation (arrow).

\section{Traumatic Dissections}

Traumatic blunt vascular injuries occur most commonly after motor vehicle accidents or other impact mechanisms that cause rapid deceleration with stretching of the internal carotid artery over the lateral masses of the cervical vertebrae. Another proposed mechanism is hyperflexion of the neck causing compression of the artery between the mandible and cervical spine. ${ }^{20}$ Vertebral dissections can occur as result of excessive rotation, distraction, or flexion-extension injuries, and are often associated with fractures extending into the transverse foramen or facet joint dislocations. ${ }^{17}$ Iatrogenic dissections are uncommon complications of cerebral angiography or interventional procedures, and occur from direct mechanical trauma to the endothelium from guidewires or catheters. ${ }^{21}$ Arterial dissection can be a dynamic process with rapid evolution of the angiographic appearance, and the intramural hematoma may track along the extracranial portion of the carotid artery, but usually stops when the artery becomes fixed as it enters the skull base (Figure 3).

\section{Presenting Symptoms, Diagnosis, and Clinical Course}

Spontaneous carotid dissections typically present with unilateral headache or neck pain which may be accompanied by a partial Horner's syndrome with ptosis and miosis, followed hours or days later by symptoms of transient cerebral or retinal ischemia or stroke, most often in the territory of the middle cerebral artery. Only about $20 \%$ of patients have an ischemic stroke without any preceding warning signs. ${ }^{3}$ Vertebral dissections may present with pain at the back of the neck or suboccipital headache, followed by ischemia in the posterior circulation. The initial manifestations of vertebral artery
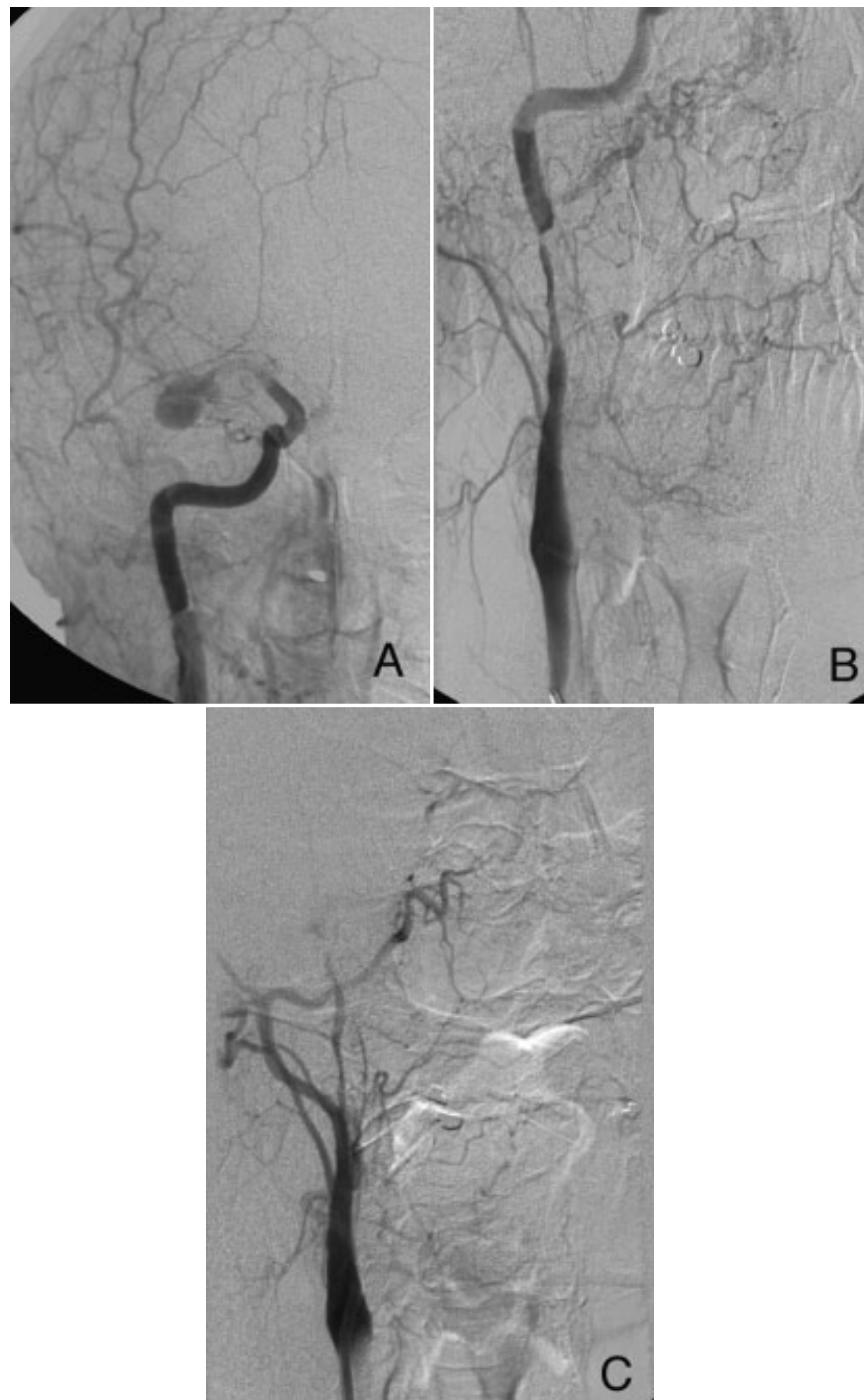

Figure 3: Anterior-posterior right carotid angiogram in a woman presenting with subarachnoid hemorrhage. A: The first run shows delay of contrast flow and only partial filling of the cerebral circulation. A right middle cerebral artery bifurcation aneurysm is identified. B: Second angiographic run, 5 minutes later, shows progressive stenosis due to expansion of the intramural hematoma. C: Third angiographic run, 15 minutes after the original series, shows extension of the dissection to the level of the skull base and near occlusion of the right internal carotid artery. The patient became acutely symptomatic with left hemiplegia. 
dissection are less stereotyped than carotid dissection, and may be interpreted as musculoskeletal in nature. Transient ischemic symptoms are less common after vertebral dissection, and stroke involving the brainstem, cerebellum, thalamus, or occipital lobes are most typical. Isolated ischemia of the cervical cord is rare. ${ }^{3}$ The incidence of cerebral ischemia caused by dissections arising spontaneously or after very minor trauma is unknown. A population-based study of all patients diagnosed with spontaneous cervical artery dissections between 1987 and 2003 found that transient ischemic attacks or stroke were presenting symptoms in $67 \% .{ }^{1}$ With the introduction of non-invasive vascular imaging leading to diagnosis in more patients with less obvious manifestations, the frequency of stroke as a presenting symptom has decreased. ${ }^{3}$ The prognosis of stroke is related to the severity of the initial ischemic insult and the extent of the collateral circulation.

Intra-arterial angiography has been the traditional standard for diagnosis of cervical dissections. However, angiography is not without risk and it is increasingly being replaced by noninvasive imaging modalities, both for primary diagnosis and follow-up. Dissection is confirmed when angiography shows an intimal flap or double lumen. Other angiographic findings suggestive of dissection include flame-shaped or tapered narrowing, "string sign", aneurysm formation, or occlusion. An MR image and MR angiography can demonstrate the luminal abnormalities seen with intra-arterial studies, but in addition can provide cross-sectional images that provide direct visualization of an intramural hematoma, characterized by a crescent-shaped hyperintense area expanding the vessel diameter around a narrowed, eccentric flow void representing the residual lumen. Magnetic resonance angiography can also be combined with standard brain imaging as well as perfusion-diffusion sequences to identify areas of infarction or potentially reversible ischemia. Multisection CT angiography provides high resolution images of the arterial wall and vessel lumen and, in the setting of trauma, can also demonstrate the relationship of arterial injuries to bone structures of the cervical spine and skull base. Routine brain imaging and CT perfusion scans can also be used to identify reversible and permanent ischemia. In the setting of trauma, CT is generally more readily accessible than MRI and the vascular imaging studies can be combined with routine trauma imaging protocols. $^{12}$

Healing of arterial dissections occurs within three to six months, with resolution of stenosis seen in $90 \%$, and recanalization of occlusions in as many as 50\%. Dissecting aneurysms resolve completely on follow-up imaging in $5-40 \%$, decrease in size in $15-30 \%$, and remain unchanged in $50-65 \%$. Resolution is more common in vertebral dissections than in carotid dissections. ${ }^{1-3,22-24}$ Aneurysm enlargement occurs rarely. Conservative management with antithrombotic therapy has been the most common approach and long term follow-up studies suggest that even in the presence of a persistent aneurysm the risk of delayed embolic events is very low. ${ }^{22-24}$

Blunt trauma causing vascular injuries in the neck is often initially occult and devastating neurological symptoms may be delayed for hours or days. It has only recently become clear that these injuries are more common than previously appreciated, and that disability secondary to cerebrovascular ischemia can be prevented by early medical intervention. In large series as many as $50 \%$ of trauma victims experienced the onset of stroke more than 24 hours after the blunt injury, presenting a window of opportunity in which screening could be applied to the at-risk patient to identify and treat the injury before the onset of complications. ${ }^{16,25}$ These advances in recognition and treatment have resulted in aggressive screening protocols and early therapy. ${ }^{14-19}$

A retrospective review of all trauma patients at the University of British Columbia prior to the onset of screening found that blunt vascular neck injuries with carotid or vertebral dissection was identified in $0.1 \%$ of all blunt trauma patients. Less than half of the dissections were suspected on the basis of presenting symptoms or pattern of injury. In those with initially occult dissections, there was a $67 \%$ delayed stroke rate and $25 \%$ mortality. ${ }^{18}$ Subsequent reports have shown that screening with angiography or non-invasive vascular imaging increases the identification of these injuries, with carotid or vertebral dissection found in approximately $1 \%$ of all trauma admissions. ${ }^{14,19,25}$ A CT angiography screening protocol applied to 1,313 consecutive blunt trauma patients at the same institution identified 170 patients with clinical criteria for vascular imaging. These criteria included all blunt trauma patients with an admission Glasgow Coma Score of 13 or less, cervical spine injury, basal skull fracture, LeForte facial fracture, significant thoracic injury, or any neurological deficit not explained by the admission CT scan. There were 33 abnormal CT angiograms, leading to catheter angiography in 23 patients. Nineteen dissections were confirmed with imaging, for an overall incidence of carotid or vertebral injuries in $1.4 \%$ of the entire trauma population. Sixty-one percent of the injuries involved the vertebral artery and 39\% involved the carotid artery. In this series, with early diagnosis and intervention consisting of anticoagulation, antiplatelet therapy, or stenting, there was one delayed stroke in a patient who did not undergo initial screening in violation of the protocol, and no deaths attributable to the vascular injury. No strokes occurred in patients who did not meet screening criteria. The overall mortality of patients with vascular injuries was $10.5 \% .^{25}$

Biffl and colleagues have proposed a grading scale with prognostic and therapeutic implications for blunt carotid injuries. ${ }^{26}$ This classification is based on the angiographic appearance of the lesion. Grade I injuries are defined as irregularity of the vessel wall or dissection with less than $25 \%$ stenosis. Grade II injuries include those with intraluminal thrombus or a raised intimal flap, or dissections with intramural hematoma causing greater than $25 \%$ stenosis. Dissecting aneurysms are classified as Grade III and complete occlusions as Grade IV injuries. Grade V injuries are those associated with complete vessel transection and evidence of free contrast extravasation. In their series, $61 \%$ of injuries were Grade I, $17 \%$ were Grade II, $15 \%$ were Grade III, $5 \%$ were Grade IV, and $4 \%$ were Grade V. Severe head injuries were evenly distributed across the injury grades, and consequently no difference in overall neurologic outcome was found. However, the incidence of delayed stroke increased with injury grade, from $3 \%$ with Grade I injuries to $44 \%$ for Grade IV. All patients with Grade V injuries died. Mortality in the entire series was $17 \%$, and $32 \%$ of the survivors suffered permanent severe neurological disability.

In the same series, follow-up angiograms were obtained in $63 \%$ of the patients. Grade I injuries went on to complete 
resolution in $70 \%$ of cases. Twenty percent of Grade II injuries resolved or improved, while $80 \%$ persisted or worsened, with $75 \%$ developing aneurysms and $12.5 \%$ progressing to occlusion. Only $4 \%$ of Grade III injuries (traumatic dissecting aneurysms) went on to heal and none of the Grade IV injuries (complete occlusion) that had repeat angiography recanalized. ${ }^{26}$

\section{Treatment}

Selection of appropriate therapy for carotid and vertebral artery dissection is dependent upon the presenting symptoms, imaging, and associated clinical factors. Arterial dissection can cause ischemic stroke either by thromboemboli forming at the site of injury or as a result of hemodynamic insufficiency due to severe stenosis or occlusion. Available evidence strongly favors embolism as the most common cause..$^{5,6,9}$ In patients with symptoms and imaging consistent with this mechanism, and in whom there is no contraindication to antithrombotic therapy, anticoagulation or antiplatelet medications are the current standard of care for spontaneous or traumatic dissections. ${ }^{6-11,27}$ No randomized clinical trial data are available. Based on the available evidence, the Cervical Artery Dissection in Ischemic Stroke Patients (CADISP) Study Group ${ }^{28}$ has recommended antiplatelet therapy in most patients with cervical dissection, noting that anticoagulation may be favored in those patients with occlusion or pseudo-occlusion of the dissected artery, recurrent events while on antiplatelet therapy, or those with free-floating intraluminal thrombus on vascular imaging.

In the prospective Canadian study of 116 patients followed for one year, $17(15 \%)$ had recurrent transient ischemic attacks, stroke, or death, mainly in the first few weeks after the dissection. Traumatic dissections were excluded, and management was at the discretion of the individual investigators. Of 105 patients with complete follow-up, the event rate in those treated with anticoagulation was $8.3 \%$ and in those treated with aspirin the event rate was $12.4 \%$, a nonsignificant difference of $4.1 \% .^{11}$ The magnitude of the potential benefit of anticoagulation may be limited and could potentially be offset by bleeding complications. ${ }^{7,8}$ A clinical trial with the power to demonstrate a $5 \%$ absolute risk reduction would require almost 2,000 patients, and is unlikely to be undertaken. ${ }^{9,11}$ A recent Cochrane review found that there is no evidence to support the routine use of anticoagulation, and the available evidence does not reliably establish whether or not anticoagulation is better than antiplatelet drugs in patients with dissection. ${ }^{9}$

In patients with evidence of hemodynamic insufficiency due to severe stenosis or occlusion, antithrombotic therapy is unlikely to be of benefit and augmentation of cerebral blood flow is required on an urgent basis. Medical management with induced hypertension and hypervolemia can be instituted in patients without cardiorespiratory compromise and, if symptoms persist, intervention aimed at restoration of normal vessel diameter to improve cerebral perfusion may be considered. Those lesions located near the skull base are particularly hazardous to approach and difficult to repair surgically. Extensive exposures necessary to achieve adequate proximal and distal vessel control may result in significant cranial nerve damage. If the injured vessel is inaccessible, proximal occlusion or segmental isolation are the most common approaches. ${ }^{29}$ Reconstruction with an in situ vein graft or extracranial to

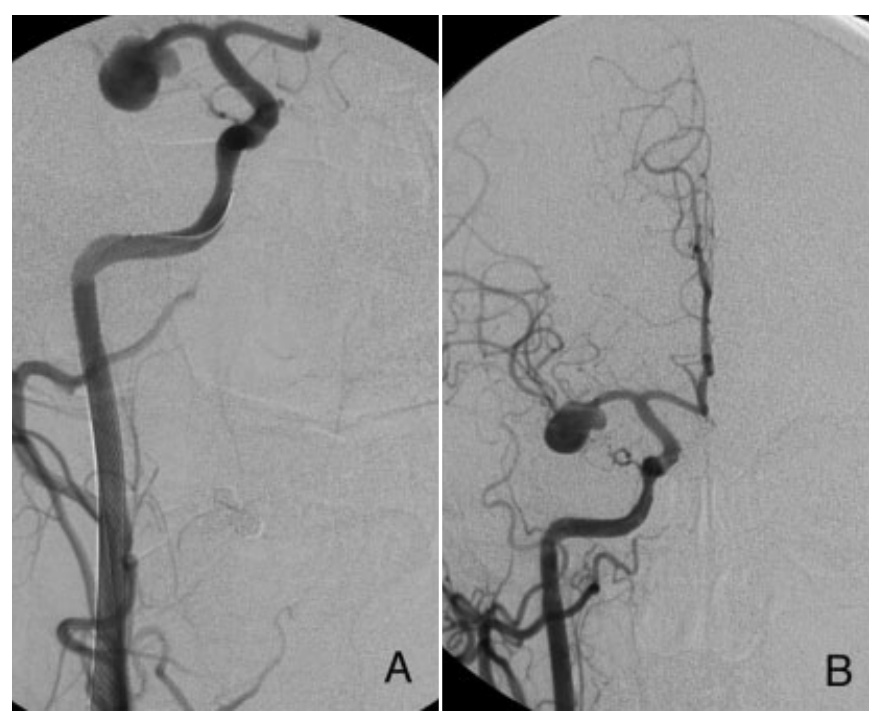

Figure 4: Same patient as Figure 3. Anterior-posterior cervical (A) and cranial $(B)$ right carotid angiograms after emergency stenting with immediate restoration of normal vessel diameter and cerebral perfusion. The patient experienced rapid recovery of her neurological deficits. Craniotomy and microsurgical clipping of the aneurysm was performed and the patient had an excellent outcome, with no residual neurological deficits.

intracranial bypass may be technically feasible but carry significant risk of morbidity. In the largest reported series of patients with carotid dissection treated surgically, Müller et al ${ }^{29}$ reported a stroke rate of $10 \%$, and cranial nerve injury in $58 \%$ of 48 patients. Ten patients experienced early post-operative vein graft occlusion. In the setting of acute cerebral ischemia, temporal and logistical considerations are additional factors that make surgical repair less attractive.

Recently, there has been considerable interest in the application of endovascular stents and covered stent grafts for the treatment of carotid and vertebral dissections and penetrating injuries. These devices permit minimally invasive arterial repairs to be performed from easily accessible sites, remote from the area of arterial injury, without compromising arterial flow. ${ }^{30-34}$ Depending on the level of injury and the diameter of the vessel involved, stenotic or occlusive dissections can be opened with standard porous carotid or coronary stents (Figure 4). Covered stent grafts may be used for expanding or symptomatic dissecting aneurysms, or those that do not resolve with medical therapy (Figure 5). Stent placement is associated with a risk of early or late thromboembolic complications or occlusion and requires periprocedural anticoagulation and continuation of single or combination antiplatelet therapy for several weeks subsequently. Kadhkodayan et $\mathrm{al}^{34}$ reported their experience with 26 patients undergoing stenting for carotid dissection. There were no procedural strokes but two patients experienced occlusion of the treated vessel, one at 22 days and one at three months after stent implantation. 


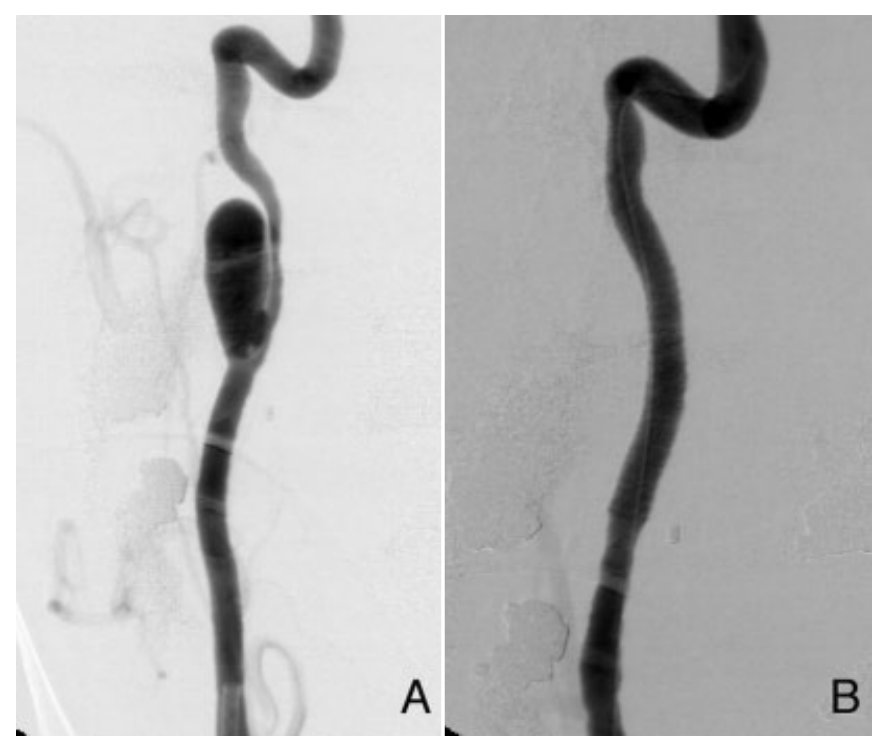

Figure 5: A: Right internal carotid angiogram showing dissecting aneurysm that had progressively increased in size on serial imaging studies. B: Angiogram following endovascular repair with a covered stent graft.

Endovascular management of dissections remains controversial. Although patients who have recurrent symptoms while on medical therapy are sometimes considered for angioplasty and stent placement, there have been no well-designed trials to support this practice, and the clinical course of most patients who avoid acute ischemic complications is relatively benign. Kremer et $\mathrm{al}^{13}$ prospectively studied the natural history of spontaneous dissection resulting in persistent stenosis or occlusion. Antithrombotic therapy was given according to the preference of the treating physician. Cases with permanent carotid stenosis or occlusion had annual rates of $0.7 \%$ for ipsilateral carotid territory stroke and $1.4 \%$ for any stroke.

The diagnosis and treatment of blunt vascular injuries affecting the extracranial carotid and vertebral arteries has evolved over the past decade, with efforts directed at identification and treatment of these injuries during the "silent period", before the onset of stroke..$^{22}$ Cothren et al ${ }^{35}$ reported a series of 15,767 bunt trauma patients in whom 727 met criteria for vascular imaging. Dissections were confirmed in 244 patients ( $34 \%$ screening yield). Of 235 who had no symptoms of dissection, 187 were treated with heparin or antiplatelet agents. In this group, one patient $(0.5 \%)$ had a stroke. Based on the estimated stroke incidence by injury grade ${ }^{26}$ an estimated 32 ischemic neurological events were averted. Of the 48 patients who were initially asymptomatic and in whom antithrombotic therapy was not initiated due to other injuries, $10(21 \%)$ had a stroke. Overall mortality for patients with traumatic dissection was $7 \%$ for those without ischemic stroke and $32 \%$ for those in whom stroke occurred. This difference was not likely a result of associated inuries, since the Injury Severity Scores were not significantly different between those with and without stroke.

\section{SUMMARY}

Dissection of the extracranial carotid and vertebral arteries is increasingly recognized and is a common cause of stroke in young patients. In the absence of randomized clinical trial data, treatment considerations must be made on the basis of the best available evidence on a patient-specific basis. Most ischemic cerebral symptoms arise from thromboembolic mechanisms and are adequately treated with anticoagulation or antiplatelet medications. Follow-up noninvasive vascular imaging with MRA or CTA should be used to document healing of the dissection before cessation of antithrombotic therapy. In the case of persistent aneurysms, antiplatelet agents should be continued indefinitely. Expanding and symptomatic dissecting aneurysms may be considered for covered stent graft repair, but in light of the relatively benign long-term outcomes of most dissections treated medically, it is important to proceed with caution when considering stent placement. The uncommon patient presenting with acute hemodynamic insufficiency should be managed with measures to increase cerebral blood flow such as keeping the head of the bed flat, volume expansion, and induced hypertension. In this setting, emergency stent placement to restore cerebral perfusion may be considered, provided that irreversible infarction has not already occurred.

There is evidence that early recognition and treatment of blunt injuries to the carotid and vertebral arteries before the onset of ischemic neurological events can lead to improved outcomes. Aggressive screening using standardized protocols and treatment with anticoagulation or antiplatelet agents has been shown to reduce the incidence of stroke in the setting of blunt trauma. ${ }^{14-}$ 21,25-27,35 Identification and treatment of these injuries prior to the development of irreversible cerebral ischemia should be a priority and demands a high degree of suspicion in patients with high risk mechanisms and injury patterns.

\section{REFERENCES}

1. Lee V, Brown R, Mandrekar J, Mokri B. Incidence and outcome of cervical artery dissection; a population-based study. Neurology. 2006;67:1809-12.

2. Caplan L, Biousse V. Cervicocranial arterial dissections. J Neuroophthalmol. 2004;24:299-305.

3. Schievink W. Spontaneous dissection of the carotid and vertebral arteries. N Engl J Med. 2001;344:898-906.

4. Dziewas R, Konrad C, Drager B, Evers S, Besselmann M, Ludemann $\mathrm{P}$, et al. Cervical artery dissection - clinical features, risk factors, therapy and outcome in 126 patients. J Neurol. 2003;250:1179-84.

5. Rubinstein S, Peerdeman S, van Tulder M, Riphagen I, Haldeman S. A systematic review of the risk factors for cervical artery dissection. Stroke. 2005;36:1575-80.

6. Norris J. Extracranial arterial dissection - anticoagulation is the treatment of choice: for. Stroke. 2005;36:2041-2.

7. Lyrer P. Extracranial arterial dissection - anticoagulation is the treatment of choice: against. Stroke. 2005;36:2042-3.

8. Georgiadis D, Caso V, Baumgartner R. Acute therapy and prevention of stroke in spontaneous carotid dissection. Clin Exp Hypertens. 2006;28:365-70.

9. Lyrer P, Engelter S. Antithrombotic drugs for carotid artery dissection. Stroke. 2004;35:613-4. 
10. Lyrer P, Engelter S. Antithrombotic drugs for carotid artery dissection (Cochrane Review). Oxford, UK: Cochrane Library;2006; Volume 1.

11. Beletsky V, Nadareishvili Z, Lynch J, Shuaib A, Woolfenden A, Norris J. Cervical arterial dissection; time for a therapeutic trial? Stroke. 2003;34:2856-60.

12. Flis C, Jager H, Sidhu P. Carotid and vertebral artery dissections: clinical aspects, imaging features and endovascular treatment. Eur Radiol. 2007;17:820-34.

13. Kremer C, Mosso M, Georgiadis D, Stockli E, Benninger D, Arnold M, et al. Carotid dissection with permanent and transient occlusion or severe stenosis: long term outcome. Neurology. 2003;60:271-5.

14. Biffl W, Moore E, Elliott J, Ray C, Offner P, Franciose R, et al. The devastating potential of blunt vertebral artery injuries. Ann Surg. 2000;231:672-81.

15. Carillo E, Osborne D, Spain D, Miller F, Senler S, Richardson J. Blunt carotid artery injuries: difficulties with the diagnosis prior to the neurologic event. J Trauma. 1999;46:1120-5.

16. Fabian T, Patton J, Croce M, Minard G, Kudsk K, Pritchard F. Blunt carotid injury: the importance of early diagnosis and anticoagulant therapy. Ann Surg. 1996;223:513-25.

17. Inamasu J, Guiot B. Vertebral artery injury after blunt cervical trauma: an update. Surg Neurol. 2006;65:238-46.

18. McKevitt E, Kirkpatrick A, Vertesi L, Granger R, Simons R. Blunt vascular neck injuries: diagnosis and outcomes of extracranial vessel injury. J Trauma. 2002;53:472-6.

19. Miller P, Fabian T, Bee T, Timmons S, Chamsuddin A, Finkle R, et al. Blunt cerebrovascular injuries: diagnosis and treatment. J Trauma. 2001;51:279-86.

20. Kraus R, Bergstein J, DeBord J. Diagnosis, treatment, and outcome of blunt carotid arterial injuries. Am J Surg. 1999;178:190-3.

21. Cloft H, Jensen M, Kallmes D, Dion J. Arterial dissections complicating cerebral angiography and cerebrovascular interventions. Am J Neuroradiol. 2000;21:541-5.

22. Benninger D, Gandjour J, Georgiadis D, Stockli E, Arnold M, Baumgartner R. Benign long-term outcome of conservatively treated cervical aneurysms due to carotid dissection. Neurology. 2007;69:486-7

23. Guillon B, Brunereau L, Biousse V, Djouhri H, Levy C, Bousser M-G. Long-term follow-up of aneurysms developed during extracranial internal carotid artery dissection. Neurology. $1999 ; 53: 117-22$
24. Touzé E, Randoux B, Meary E, Arquizan C, Meder J, Mas J. Aneurysmal forms of cervical artery dissection - associated factors and outcome. Stroke. 2001;32:418-23.

25. Schneidereit N, Simons R, Nicolau S, Graeb D, Brown D, Kirkpatrick A, et al. Utility of screening for blunt vascular neck injuries with computed Tomographic angiography. J Trauma. 2006;60:209-16.

26. Biffl W, Moore E, Offner P, Brega K, Franciose R, Burch J. Blunt carotid injuries: implications of a new grading scale. J Trauma. 1999;45:845-66.

27. Cothren C, Moore E, Biffl W, Ciesla D, Ray C, Johnson J, et al. Anticoagulation is the gold standard therapy for blunt carotid injuries to reduce stroke rate. Arch Surg. 2004;139:540-6.

28. Engelter S, Brandt T, Debette S, Caso V, Lichy C, Pezzini A, et al. Antiplatelets versus anticoagulation in cervical artery dissection. Stroke. 2007:38:2605-11.

29. Müller B, Luther B, Hort W, Neumann-Haefelin T, Aulich A, Sandmann W. Surgical treatment of 50 carotid dissections: indications and results. J Vasc Surg. 2000;31:980-8.

30. Redekop G, Marotta T, Weill A. Treatment of traumatic aneurysms and arteriovenous fistulas of the skull base by using endovascular stents. J Neurosurg. 2001;95:412-9.

31. Cohen J, Leker R, Gotkine M, Gomori M, Ben-Hur T. Emergency stenting to treat patients with carotid artery dissection clinically and radiologically directed therapeutic decision making. Stroke. 2003;34:254-7.

32. Fateri F, Groebli Y, Rüfenacht D. Intra-arterial thrombolysis and stent placement in the acute phase of blunt internal carotid artery trauma with subocclusive dissection and thromboembolic complication: case report and review of the literature. Ann Vasc Surg. 2005;19:1-4.

33. Edgell R, Abou-Chebl A, Yadav J. Endovascular management of spontaneous carotid artery dissection. J Vasc Surg. 2005;42: 854-60.

34. Kadkhodayan Y, Jeck D, Moran C, Derdeyn C, Cross D. Angioplasty and stenting in carotid dissection with or without associated pseudoaneurysm. Am J Neuroradiol. 2005;26: 2328-35.

35. Cothren C, Moore E, Ray C, Ciesla D, Johnson J, Moore J, et al. Screening for blunt cerebrovascular injuries is cost-effective. Am J Surg. 2005;190:849-54 